\title{
Changes in nasotracheal tube depth in response to head and neck movement in children.
}

\author{
Masanori Tsukamoto1, Hitoshi Yamanaka2, Takashi Hitosugi2, Takeshi Yokoyama2
}

\author{
1Department of Dental Anesthesiology, Kyushu University Hospital, Fukuoka, Japan \\ 2Department of Dental Anesthesiology, Faculty of Dental Science, Kyushu University, Fukuoka, Japan
}

\begin{abstract}
$\langle$ Background $\rangle$
The position of the tube tip is important to avoid accidental extubation and tracheobroncheal intubation especially in pediatric cases. The position of tube tip may change relatively in the trachea by moving the head position. Therefore, we should pay attention to the relation of the vocal cords and the cuff end, as tracheal tube with cuff is often inserted via the nasal cavity for dental surgery even in pediatric cases. Head movement may cause changes in the length of the trachea (t-length) and/or in the distance between the nare and the vocal cords (n-v-distance). We investigated the changes in t-length and n-v-distance in children undergoing nasotracheal intubation to reveal the relation between the vocal cords and the tracheal tube.
\end{abstract}

\section{$\langle$ Conclusion 〉}

Change of head position may cause positional change of the tube tip relatively in the trachea, which may increase the risk of accidental extubation, tracheobroncheal intubation and damage on the vocal cords by tube cuff. Our results showed that this positional change of the tube tip mainly depends on the change of t-length. The positional change between the vocal cords and the tube was not significant. Therefore, we need not too anxious about accidental extubation and cuff damage on the vocal cords.

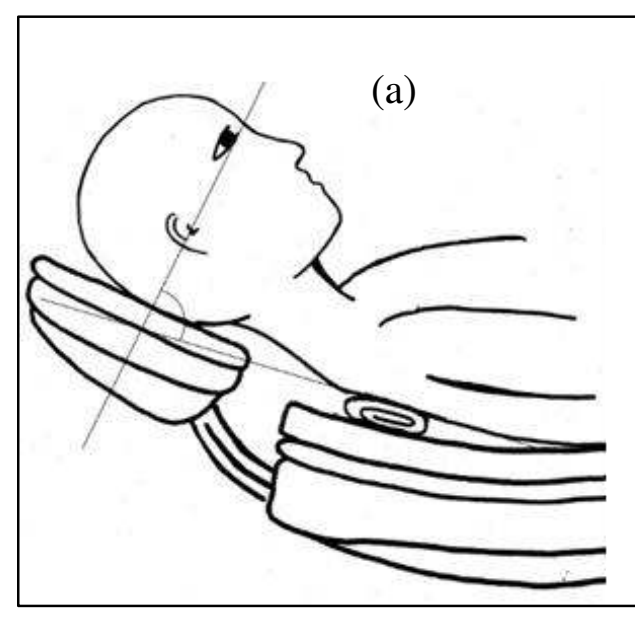

(a) Flexion: $80^{\circ}$

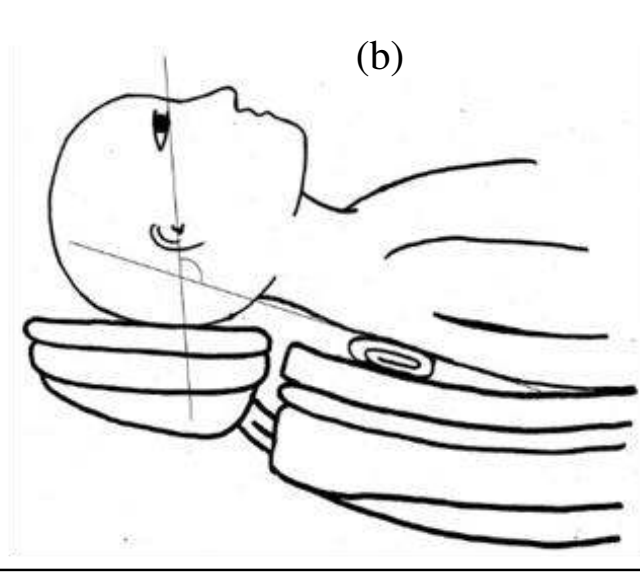

Fig.1 Head positions (b) Neutral: $110^{\circ}$

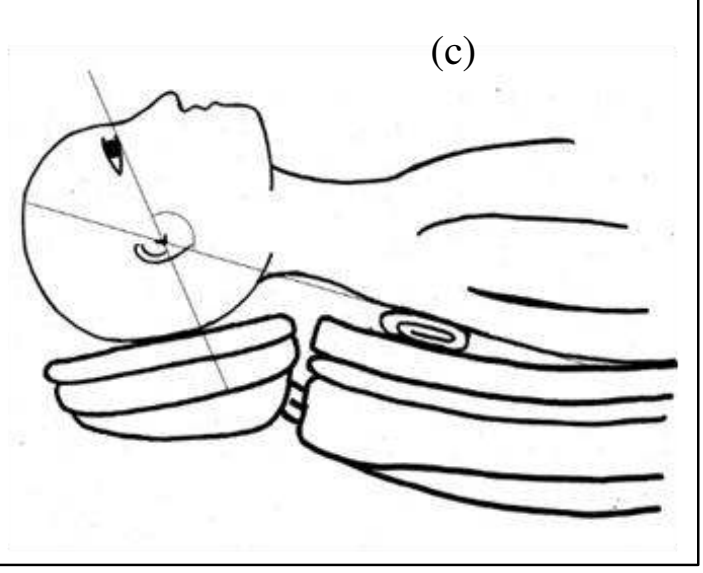

(c) Extension: $130^{\circ}$

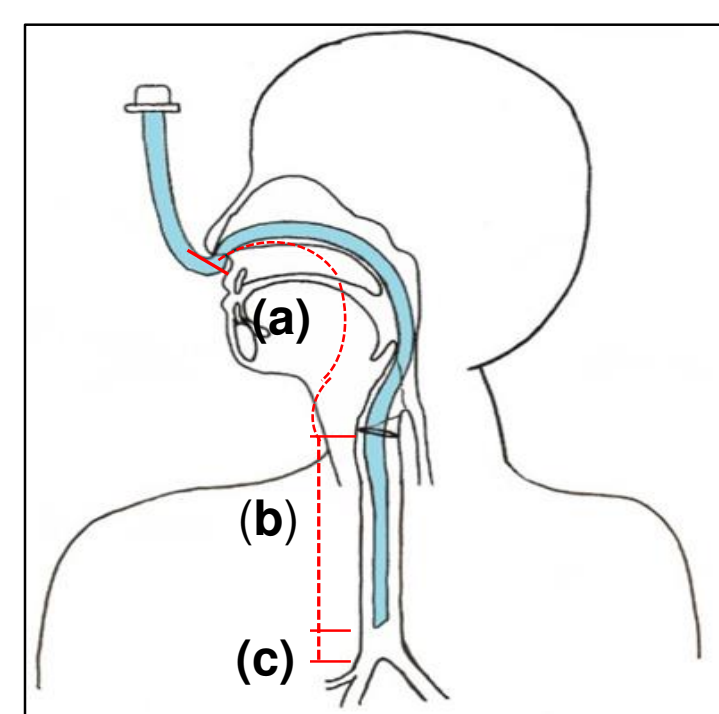

Fig.2

a: distance between the nare and vocal cords (n-v-distance)

b: distance between the vocal cords and the tip of the tracheal tube

c: distance between the tip of the tracheal tube and the carina

\begin{tabular}{|l|c|c|c|c|}
\hline $\begin{array}{l}\text { Tube size ID } \\
(\mathbf{m m})\end{array}$ & $\begin{array}{c}\mathbf{4 . 5} \\
(\mathbf{n = 2 0})\end{array}$ & $\begin{array}{c}\mathbf{5 . 0} \\
(\mathbf{n = 2 0})\end{array}$ & $\begin{array}{c}\mathbf{5 . 5} \\
(\mathbf{n = 2 0})\end{array}$ & $\begin{array}{c}\mathbf{6 . 0} \\
(\mathbf{n = 2 0})\end{array}$ \\
\hline male/female $(\mathrm{n})$ & $6 / 14$ & $9 / 11$ & $13 / 7$ & $14 / 6$ \\
\hline Age $(\mathrm{yrs})$ & $3.4 \pm 0.7$ & $4.8 \pm 1.0$ & $5.9 \pm 0.6$ & $7.0 \pm 1.1$ \\
\hline Height $(\mathrm{cm})$ & $92.6 \pm 4.7$ & $101.6 \pm 5.2$ & $111.1 \pm 3.7$ & $118.5 \pm 5.2$ \\
\hline Weight $(\mathrm{kg})$ & $13.4 \pm 1.2$ & $16.5 \pm 2.3$ & $19.3 \pm 1.6$ & $22.2 \pm 2.8$ \\
\hline BMI $\left(\mathrm{kg} / \mathrm{m}^{2}\right)$ & $15.7 \pm 0.8$ & $15.9 \pm 1.3$ & $15.6 \pm 1.3$ & $15.8 \pm 1.3$ \\
\hline
\end{tabular}

Table 1 Patient characteristics

$\langle$ Method

The study protocol was approved by the Ethics Committee of Kyushu University Hospital (approval number 28-145).

Patients: aged 2-15 y undergoing dental surgery.

Anesthetic management: After nasotracheal intubation with an uncuffed nasotracheal tube $(4.5-6.0 \mathrm{~mm})$, the tube was fixed at the patient's nares. The distance between the tube tip and the first carina was measured using a fibrescope with neutral head position; the angle between the Frankfort plane and horizontal plane set was $110^{\circ}$. The location of the tube in relation to the vocal cords was then evaluated. These measurements were repeated at angles of $80^{\circ}$ (flexion) and $130^{\circ}$ (extension).

Measurement: The t-length, $n$-v-distance and the distance between the vocal cords and tracheal tube tip were then calculated using the results of these measurements.

Statistical analyses: In the univariate analysis, parametric data were analysed using the Kruskal-Wallis test and SteelDwass multiple comparisons.

\begin{tabular}{|c|c|c|c|c|c|}
\hline & & $\begin{array}{l}\text { Flexion } \\
\left(80^{\circ}\right)\end{array}$ & $\begin{array}{l}\text { Neutral } \\
\left(110^{\circ}\right)\end{array}$ & $\begin{array}{l}\text { Extension } \\
\left(130^{\circ}\right)\end{array}$ & $P$ value \\
\hline \multirow{3}{*}{$\begin{array}{l}\text { ID } 4.5 \\
(\mathrm{n}=20)\end{array}$} & $\mathrm{n}$-v-distance $(\mathrm{mm})$ & $116.8 \pm 7.2$ & $119.2 \pm 7.0$ & $120.3 \pm 7.0$ & 0.299 \\
\hline & $\begin{array}{l}\text { distance between tracheal tube tip and } \\
\text { carina }(\mathrm{mm})\end{array}$ & $19.2 \pm 6.1$ & $25.9 \pm 7.1$ & $32.7 \pm 7.8$ & $0.001>*$ \\
\hline & t-length $(\mathrm{mm})$ & $73.1 \pm 7.0$ & $77.4 \pm 7.6$ & $83.1 \pm 6.5$ & $0.001>*$ \\
\hline \multirow{3}{*}{$\begin{array}{l}\text { ID 5.0 } \\
(\mathrm{n}=20)\end{array}$} & $\mathrm{n}$-v-distance $(\mathrm{mm})$ & $123.0 \pm 9.3$ & $125.1 \pm 10.1$ & $126.1 \pm 10$ & 0.453 \\
\hline & $\begin{array}{l}\text { distance between tracheal tube tip and } \\
\text { carina }(\mathrm{mm})\end{array}$ & $23.0 \pm 10.4$ & $30.6 \pm 10.6$ & $36.0 \pm 11.1$ & $0.001>*$ \\
\hline & $\mathrm{t}$-length $(\mathrm{mm})$ & $79.8 \pm 7.5$ & $85.2 \pm 6.7$ & $89.6 \pm 7.1$ & $0.001 *$ \\
\hline \multirow{3}{*}{$\begin{array}{l}\text { ID 5.5 } \\
(\mathrm{n}=20)\end{array}$} & $\mathrm{n}$-v-distance $(\mathrm{mm})$ & $129.9 \pm 9.7$ & $133.3 \pm 10.2$ & $134.6 \pm 9.9$ & 0.233 \\
\hline & $\begin{array}{l}\text { distance between tracheal tube tip and } \\
\text { carina }(\mathrm{mm})\end{array}$ & $30.2 \pm 11.1$ & $38.3 \pm 9.8$ & $45.0 \pm 9.6$ & $0.003 *$ \\
\hline & $\mathrm{t}$-length $(\mathrm{mm})$ & $86.0 \pm 8.2$ & $90.3 \pm 7.3$ & $95.8 \pm 7.0$ & $0.001 *$ \\
\hline \multirow{3}{*}{$\begin{array}{l}\mathrm{ID} 6.0 \\
(\mathrm{n}=20)\end{array}$} & $\mathrm{n}$-v-distance (mm) & $136.8 \pm 8.7$ & $139.2 \pm 8.5$ & $140.9 \pm 8.6$ & 0.153 \\
\hline & $\begin{array}{l}\text { distance between tracheal tube tip and } \\
\text { carina }(\mathrm{mm})\end{array}$ & $34.4 \pm 10.0$ & $41.2 \pm 9.9$ & $47.7 \pm 9.7$ & $0.001>*$ \\
\hline & t-length $(\mathrm{mm})$ & $92.7 \pm 8.8$ & $97.0 \pm 9.0$ & $102.1 \pm 8.7$ & $0.002 *$ \\
\hline
\end{tabular}

Table. 2 The values are shown as the means \pm standard deviation. $* \mathrm{P}<0.05$,

Kruskal-Wallis test and Steel-Dwass multiple comparison.

$\langle$ Results and Discussion 〉

In the case of forward bending of the neck, the t-length changed significantly from $84.5 \pm 10.8 \mathrm{~mm}$ to $80.0 \pm 10.8 \mathrm{~mm}(\mathrm{p}=0.004)$. The $\mathrm{n}$ - $\mathrm{v}$-distance changed from $125.6 \pm 12.0 \mathrm{~mm}$ to $123.0 \pm 11.7 \mathrm{~mm}(\mathrm{p}=0.179)$. In the case of extension, the $\mathrm{t}-$ length changed significantly to $88.7 \pm 10.4 \mathrm{~mm}(\mathrm{p}=0.005)$. The $\mathrm{n}$ - $\mathrm{v}$-distance changed to $126.9 \pm 12.2 \mathrm{~mm}(\mathrm{p}=0.501)$. The change of t-length was significantly larger than that of $\mathrm{n}$-v-distance. These data suggest that the change of the position of the tube tip depends mainly on the change in the t-length. When the tube with cuff is used, we may need not be too nervous for the change of tube position to the vocal cords. 